\title{
A Comprehensive Analysis of Potential Gastric Cancer Prognostic Biomarker ITGBL1 Associated With Immune Infiltration and Epithelial- mesenchymal Transition
}

\section{Zhe Wang}

Tongji Hospital, Tongji University School of Medicine

\section{Liu Fu}

Putuo people's hospital, Tongji University

\section{Junjie Zhang}

Tongji Hospital, Tongji University School of Medicine

Yanli Ge

Tongji Hospital, Tongji University School of Medicine

Cheng Guo

Tongji Hospital, Tongji University School of Medicine

Rui Wang

Tongji Hospital, Tongji University School of Medicine

Min Deng

the first affiliated hospital of bengbu medical college

Qizhi Wang

the first affiliated hospital of bengbu medical college

Zhirong Wang ( $\nabla$ wangzr92989@126.com )

Tongji Hospital, Tongji University School of Medicine

\section{Research}

Keywords: epithelial-mesenchymal transition, immune infiltration, gastric cancer, ITGBL1

Posted Date: September 21st, 2021

DOI: https://doi.org/10.21203/rs.3.rs-898472/v1

License: (c) (1) This work is licensed under a Creative Commons Attribution 4.0 International License. Read Full License 


\section{Abstract}

Background: Integrin, beta-like 1 (ITGBL1) is involved in a variety of human malignancies. However, the information on the involvement of ITGBL1 in gastric carcinoma (GC) is limited. Hence, this study aimed to further explore the functions and mechanisms of ITGBL1 in GC.

Methods: First, multiple bioinformatics databases, including Oncomine, Timer, UALCAN, and KaplanMeier Plotter, were used to predict the expression level and prognostic value of ITGBL1, as well as its association with immune infiltration and epithelial-mesenchymal transition (EMT) in GC. Quantitative reverse transcription-polymerase chain reaction and immunohistochemical analysis were used to to detect the expression of ITGBL1 in both GC tissues and cells. Then, targeted silencing of ITGBL1 in GC cells was further to examine the biological functions of ITGBL1.

Results: These databases revealed that ITGBL1 was overexpressed and affected the overall survival in GC. Besides, the expression of ITGBL1 positively correlated with immune-infiltrating cells and EMT-related markers. Subsequently, molecular biology experiments verified these predictions. In GC tissues and cells, ITGBL1 was notably overexpressed. Loss-of-function studies showed that the knockdown of ITGBL1 significantly suppressed migration and invasion but promoted apoptosis in MGC803 GC cells. Furthermore, the inhibition of ITGBL1 resulted in remarkably increased protein expression levels of cadherin 1 (CDH1), while the expression of Vimentin, Snail, and TGF- $\beta 1$ was downregulated, indicating the initiation and progression of GC caused by ITGBL1 partly via inducing EMT.

Conclusion: To sum up, the findings indicated that ITGBL1 acted as a valuable oncogenic factor in GC.

\section{Introduction}

Gastric carcinoma (GC) is the fourth most common malignant tumor and third in terms of cancer-related mortality in the world [1]. Although substantial progress has been made on the pathogenesis and molecular biology of GC, approximately $80 \%$ of patients with GC do not receive the best treatments owing to locally advanced disease and metastasis at diagnosis [2]. In addition, the traditional therapeutic methods, such as surgery and chemotherapy, have limited value in advanced disease [3]. Therefore, further exploration of GC is needed, including potential biomarkers for early diagnosis.

Integrins are an important type of cell surface receptors via mediating the adhesion between cells and extracellular matrixes to receive and conduct cascade signals and hence regulate cell survival, proliferation, movement, and other biological behaviors [4]. Increasing evidence indicates that numerous integrins are involved in the initiation and progression of tumors [5-7]. Integrin, beta-like 1 (ITGBL1), also called Ten $\beta$ Integrin EGF-like Repeat Domains (TIED), encodes beta integrin-related extracellular matrix protein, which is highly similar to integrin $\beta$ subunits. ITGBL1 was first discovered from overlapping CDNA clones in 1999 [8]. Previous studies showed that ITGBL1 was dysregulated in several tumors and acted as either carcinogenic or tumor suppressor. For instance, the ITGBL1 level was elevated in colorectal carcinoma (CRC) and accelerated cell proliferation and migration [9]. Similarly, in hepatocellular cancer, 
high ITGBL1 expression was associated with a poor prognosis [10]. In contrast, ITGBL1 expression was downregulated in non-small cell lung cancer (NSCLC) and inhibited migration and invasion via binding to miR-576-5p [11]. Li et al. reported that ITGBL1 expression was upregulated and had a high positive correlation with distant metastasis and tumor-node-metastasis stage in GC [12]. However, the functions and molecular mechanisms of ITGBL1 are still incompletely investigated in GC.

In the present study, ITGBL1 levels were first compared through multiple bioinformatics repository and found to be upregulated in GC tissues compared with those in normal tissues. Subsequently, the finding was verified by quantitative reverse transcription-polymerase chain reaction (qRT-PCR) and immunohistochemical analysis with fresh clinical samples collected. Next, we performed a comprehensive analysis including the clinical prognosis of ITGBL1 and the association between ITGBL1 expression and immune infiltration and epithelial-mesenchymal transition (EMT) using diverse public databases. Finally, functional and mechanical assays were conducted to further investigate the biological behavior of ITGBL1 and confirm the correlation between ITGBL1 and EMT. It was concluded that ITGBL1 might play a highlight role in the carcinogenicity of GC and serve as a potential marker in targeted therapy.

\section{Results}

\section{Upregulated ITGBL1 expression in GC tissues}

First, we analyzed ITGBL1 expression in various tumors using the Tumor Immune Estimation Resource (Timer) database (http://timer.cistrome.org/) [13], which revealed the dysregulation of ITGBL1 in different tumors (Fig. 1A). Subsequently, a public database Oncomine (https://www.oncomine.org/) [14] was used to investigate the levels of ITGBL1 mRNA in GC tissues. The world's largest oncogene chip database was used to analyze the data uploaded from DErrico and Cho gastric dataset samples. The results showed that mRNAs of ITGBL1 were overexpressed in both diffuse gastric adenocarcinoma and gastric intestinal-type adenocarcinoma than in normal tissues (Fig. 1B). Consistent with this, TCGA gene expression data available in an online web tool UALCAN (http://ualcan.path.uab.edu) [15] were used for analyzing cancer omics data. The change in the ITGBL1 translation level in stomach adenocarcinoma tissues was 3.5-fold relative to that in normal tissues (Fig. 1C). Subsequently, 13 paired GC tissues were collected for qRT-PCR to verify bioinformatics findings. The results indicated that ITGBL1 was often overexpressed in GC tissues compared with paired normal tissues. The ITGBL1 mRNA expression level was higher in 8 of 13 tissues, accounting for $61.5 \%$; however, only two patients had low expression in GC tissues (Fig. 1D-1F, P<0.05). Besides, immunohistochemical staining of ITGBL1 was performed in 30 paired GC tissues, revealing that the ITGBL1 protein level remarkably increased in GC tumors with $63.3 \%$ (19/33 patients) of the patients exhibiting higher ITGBL1 protein levels relative to the adjacent nontumor tissues (Fig. 1G-1H). IHC performed with these paired tissues showed more ITGBL1-positive tissues in tumor tissues than in nontumor tissues (Fig. 1I, P<0.01). 
In addition, in the public UALCAN website, various clinicopathological characteristics, including cancer stages, patient's sex, patient's age, tumor grade, Helicobacter pylori infection status, and nodal metastasis status of GC samples in the TCGA were further analyzed. The results consistently showed that the mRNA expression level of ITGBL1 was significantly higher in patients than in healthy individuals based on subgroups, including cancer stages $2-4$, sex, age 41-80 years, tumor grade $2-3, H$. pylori infection status, and nodal metastasis status with statistical significance (Fig. 2). Thus, it was concluded that ITGBL1 was abnormally expressed in various tumors including GC.

\section{Higher expression of ITGBL1 with poorer prognosis in GC}

For predicting the clinical prognosis of ITGBL1, the Kaplan-Meier Plotter (www.kmplot.com) [16] was used to analyze the overall survival (OS) in different microarray chips and RNA-seq data. Four microarray chips commonly indicated that high ITGBL1 was closely related to poor OS (Fig. 3A-3D). Consistent with this, RNA-seq results showed that upregulated ITGBL1 was significantly associated with the poor OS of patients with GC (Fig. 3E). Next, cBioPortal (www.cbioportal.org/) [17], a multidimensional cancer genomics data set, including somatic mutation, DNA copy-number alterations (CNAs), deep deletion, and mRNA upregulation, was used for deeply investigating the genomic alterations of ITGBL1 in GC. ITGBL1 had at least one alteration in 31 of $258(12 \%)$ patients with GC. Among these, mRNA upregulation accounted for $6 \%$ (Fig. 3F). Overall, these findings revealed that ITGBL1 might serve as a potential diagnostic and prognostic indicator of GC with different mutations.

\section{ITGBL1 level was associated with the number of immune-infiltrating cells in GC}

The Timer database was used to predict the relationship of the ITGBL1 level with the number of immuneinfiltrating cells in GC. The results showed that ITGBL1 was significantly positively associated with the number of CD $4+\mathrm{T}$ cells $(r=0.277, P=4.30 \mathrm{e}-08), \mathrm{CD} 8+$ cells $(r=0.438, P=3.41 \mathrm{e}-19)$, neutrophils $(r=0.27$, $P=9.15 \mathrm{e}-08)$, macrophages $(r=0.685, P=8.23 \mathrm{e}-54)$, and myeloid dendritic cells $(r=0.402, P=3.72 \mathrm{e}-16)$. However, no obvious correlation was found between the ITGBL1 level and the number of B cells $(r=0.044$, $P=3.95 \mathrm{e}-01$ ). In addition, we performed the TISIDB (http://cis.hku.hk/TISIDB/) [18] database analysis to explore the association between the ITGBL1 level and number of TILs. In GC, the number of 19 immuneinfiltrating cells positively correlated with the expression of ITGBL1. Notably, the top six cells were natural killer cells $(r=0.553, P<2.2 \mathrm{e}-16)$, mast cells $(r=0.54, P<2.2 \mathrm{e}-16)$, macrophages $(r=0.536, P<2.2 \mathrm{e}-16)$, Type $1 \mathrm{~T}$ helper cells $(r=0.472, P<2.2 \mathrm{e}-16)$, natural killer T cells $(r=0.463, P<2.2 \mathrm{e}-16)$, and regulatory $\mathrm{T}$ cells $(r=0.458, P<2.2 \mathrm{e}-16)$, which displayed a moderate relationship. Subsequently, we explored the impact of immune cells on the prognosis of ITGBL1 using the Kaplan-Meier Plotter. We found that for natural killer T cells $(P=0.037)$, regulatory T cells $(P=0.0042), \mathrm{CD} 4+$ memory T cells $(P=0.0025)$, Type 1 T helper cells $(P=0.031)$, and Type 2 T helper cells $(P=0.0062)$, higher ITGBL1 expression predicted poor OS. However, no significant correlations were found with abundant CD $8+$ T cells $(P=0.084)$, mesenchymal stem cells $(P=0.09)$, and macrophages $(P=0.074)$. Thus, our findings suggested that ITGBL1 might be involved in regulating immune infiltration and influenced the prognosis of GC partly through immune infiltration. 


\section{Silencing of ITGBL1 expression promoted GC cell MGC803 apoptosis and suppressed MGC803 cell migration and invasion in vitro}

The ITGBL1 expression levels in an immortalized normal gastric epithelial cell line GES-1 and three GC cell lines (BGC823, SGC7901, and MGC803) were compared. The results showed that ITGBL1 had relatively increased expression in all GC cell lines, especially in MGC803, with a fold change of up to 12 relative to that in GES-1 (Fig. 6A). Therefore, MGC803 was chosen for investigating the knockdown of ITGBL1. Next, three specific shRNAs targeting ITGBL1 were designed and transfected into MGC803 cells. The qRT-PCR analysis revealed that ITGBL1-Sh3 mRNA levels were successfully and effectively knocked down compared with mock (without shRNA transfection) and NC (with negative control shRNA transfection), from a baseline of 1.00 to 0.38 in MGC803 cells $(P=0.0026$, Fig. 6B). Subsequently, the ITGBL1 protein level was effectively downregulated compared with that in mock and NC cells $(P=0.0059$ and 0.0083 , respectively) in MGC803 cells (Fig. 6C). Thus, ITGBL1-Sh3 cells were remarkably constructed and used in all subsequent experiments. Flow cytometry assay was conducted to examine whether the knockdown of ITGBL1 expression caused programmed cell death. Relative to transfection with or without NC (4.37 \pm 0.48 and $3.63 \pm 0.38) \%$, the inhibition of ITGBL1 expression remarkably increased cell apoptosis in MGC803 cells ( $8.57 \pm 0.83) \%(P=0.0118$ and 0.0056 , respectively; Fig. 6D and 6E). Next, the involvement of ITGBL1 in the migration and invasion of GC cells was further characterized using Transwell assay, revealing that ITGBL1 silencing resulted in a significant decrease in the number of migrating MGC803 cells versus mock and NC cells ( $P=0.002$ and 0.0021 , respectively; Fig. 6F). Similarly, the invasion ability was inhibited by transfection with ITGBL1-Sh3 compared with transfection with NC or mock ( $P=0.0046$ and 0.0107 , respectively; Fig. $6 \mathrm{G})$.

\section{ITGBL1 involvement in EMT in GC cells}

The Timer database was used to explore the correlations between ITGBL1 and EMT-related factors including CDH1, CDH2, VIM, SNAIL, TWIST1, and TGF- $\beta 1$. The predicted results showed that ITGBL1 expression was negatively associated with $\mathrm{CDH} 1$ expression $(R=-0.17, P=9.22 \mathrm{e}-04)$ but had a positive association with $\mathrm{CDH} 2(R=0.579, P=2.92 \mathrm{e}-35)$, Vimentin $(R=0.692, P=3.11 \mathrm{e}-55)$, Snai1 $(R=0.308, P=$ 8.52e-10), TWIST1 ( $R=0.611, P=3.1 \mathrm{e}-40)$, and TGF- $\beta 1(R=0.545, P=1.09 \mathrm{e}-30)$ (Fig. 7A). Besides, another database, Starbase online server (http://www.sysu.edu.cn/) [19], was used, and the trend of relevance was consistent. The ITGBL1 level strongly positively correlated with $\mathrm{CDH} 2(R=0.481, P=4.04 \mathrm{e}-$ 23), Vimentin $(R=0.587, P=3.98 \mathrm{e}-36)$, Snai1 $(R=0.342, P=1.01 \mathrm{e}-11)$, TWIST1 $(R=0.547, P=1.08 \mathrm{e}-30)$, and TGF- $\beta 1(R=0.542, P=5.27 \mathrm{e}-30)$, but significantly negatively correlated with $\mathrm{CDH} 1(R=-0.215, P=$ 2.65e-05) (Fig. 7B). After predicting, the expression of EMT biomarkers and transcription factors was investigated using the Western blot analysis to further identify the relationship between ITGBL1 and EMT in MGC803 cells. The results showed that the inhibition of ITGBL1 led to a distinct enhancement in the expression of epithelial biomarker $\mathrm{CDH} 1$. In contrast, the expression of mesenchymal biomarker Vimentin and main transcription factors Snail and TGF- $\beta 1$ was remarkably downregulated in ITGBL1-Sh3 cells compared with mock and NC cells (Fig. 7C). The results elucidated that the inhibition of ITGBL1 in part suppressed the process of EMT. 


\section{Discussion}

Extracellular matrix (ECM) provides a suitable microenvironment for tumor survival and activity and is involved in tumor barrier function. Previous studies showed that ECM molecules such as periostin and dermatopontin were important in tumor initiation and progression [20, 21]. Recently, ITGBL1, as an ECMrelated protein, has been found to be dysregulated in malignant tumors; however, the function and biological mechanism of ITGBL1 still remains unclear. In the present study, ITGBL1 expression was upregulated in both GC tissues and GC cells. Hence, ITGBL1 might serve as an innovative prognostic biomarker for patients with GC.

Accumulating evidence indicated that ITGBL1 was crucial in carcinogenesis and cancer progression. Multiple studies showed that ITGBL1 functioned as an oncogene [22, 23]. For example, in acute myeloid leukemia (AML), ITGBL1 methylation was reported to have an impact on prognosis, and patients with hypermethylation of ITGBL1 tended to have leukemia-free survival and shorter OS [24]. Similarly, high expression of ITGBL1 facilitated cell migration and adhesion in ovarian cancer through Wnt/ planar cell polarity and focal adhesion kinase (FAK) /SRC signaling pathways. Moreover, higher expression of ITGBL1 was more likely to cause tumorigenesis and was related to poorer prognosis [25]. In contrast, ITGBL1 functioned as a tumor suppressor in NSCLC, inhibiting cell migration and invasion [11]. A previous study reported based on integrated bioinformatics analysis that ITGBL1 was a prognostic factor [26]. However, the biological mechanism of the dysfunction of ITGBL1 has not been fully understood. The present study showed using public database Timer, Oncomine, UALCAN, and clinical samples that ITGBL1 was highly expressed in GC tissues compared with adjacent normal tissues. Besides, ITGBL1 in GC tissues derived from TCGA samples was significantly highly expressed in subgroups including cancer stage, tumor grade, and lymph node metastasis compared with normal gastric tissues. In addition, higher ITGBL1 expression was closely associated with poorer OS in patients with GC, which indicated that ITGBL1 might serve as an independent prognostic factor in GC. Besides, ITGBL1 was overexpressed in GC cells in vitro, which was consistent with its expression in tissues. Silencing ITGBL1 suppressed MGC803 cell migration and invasion but promoted apoptosis. CNAs and mRNA expression in the TCGA cohort were first checked to further investigate the genomic changes of ITGBL1 in gastric carcinogenesis. Despite the amplification and mutation of ITGBL1, more cases revealed ITGBL1 mRNA expression, indicating that the post-transcriptional regulation of ITGBL1 had a pivotal role and required more attention, thus providing ideas for further research.

Immune infiltration plays a decisive role in tumorigenesis and is one of the critical mechanisms for tumor initiation and progression. Immune-infiltrating CD4 + T cells are involved in anti-tumor function, which not only contributes to the activation of $\mathrm{CD} 8+T$ cells but also helps in the generation and preservation of memory cytotoxic $\mathrm{T}$ lymphocyte responses [27]. Neutrophils promote gastric cancer progression by reducing T-cell immunity [28]. Cheli et al. [29] reported that ITGBL1, as a novel immunomodulator, promoted melanoma development by inhibiting the cytotoxicity of natural killer cells both in vitro and in vivo. In this study, we found that ITGBL1 had a close relationship with CD $4+\mathrm{T}$ cells, CD $8+$ cells, neutrophils, macrophages, and myeloid dendritic cells. Also, higher ITGBL1 expression predicted poor OS 
in enriched natural killer T cells, regulatory T cells, CD4 + memory T cells, Type $1 \mathrm{~T}$ helper cells, and Type 2 T helper cells. These findings revealed that ITGBL1 reflected the immune status of GC in part and provided notable insights into GC immunotherapy.

EMT phenotype is a transient and reversible procedure by which a polarized, epithelial phenotype switches to an elongated mesenchymal phenotype, resulting in increased migration and invasion [30]. A substantial body of evidence implied that EMT was significantly associated with metastasis and prognosis of various cancers, including GC [31]. Previous studies confirmed that the regulation of EMT was a complex network including multiple signaling pathways such as Wnt/ $\beta$-catenin, Notch, hepatocyte growth factor, and TGF- $\beta$ [32-34]. Among these, the TGF- $\beta$ signaling pathway was the major contributor, regulating a series of cellular processes including intercellular substance production, differentiation, apoptosis, immune reaction, and inflammatory response [35]. For example, the TGF- $\beta$ signaling pathway functioned as a key regulator of fibrogenesis, and elevated expression of ITGBL1 promoted HBV-related liver fibrogenesis by interacting with TGF- $\beta 1$ [36]. In CRC, ITGBL1 levels strongly correlated with EMTassociated genes, serving as a crucial indicator of an EMT phenotype [37]. High expression of ITGBL1 facilitated bone metastasis via inducing the TGF- $\beta$ signaling pathway in breast cancer [38]. Li et al. found that high expression of ITGBL1 promoted invasion and migration and activated EMT in prostate cancer [39]. The present study found that the expression levels of EMT-related biomarker $\mathrm{CDH} 1$ increased, while the expression of biomarker Vimentin and EMT transcription factor Snail decreased after the downregulation of ITGBL1. In addition, the protein expression level of EMT-stimulating factor TGF- $\beta$ decreased after the silencing of ITGBL1, suggesting that ITGBL1 could promote EMT in part by activating the TGF- $\beta$ signaling pathway in GC. In addition, the online Timer and Starbase analysis yielded consistent results that ITGBL1 expression correlated negatively with $\mathrm{CDH} 1$ expression, but correlated positively with Vimentin, Snai1, TWIST1 and TGF- $\beta 1$ expression. These results further demonstrated that ITGBL1 regulated the biological functions of GC partly via inducing EMT signaling pathways.

\section{Conclusion}

In conclusion, based on public bioinformatics analysis and biological experiments, ITGBL1 was predicted and found to be highly expressed in GC and associated with immune infiltration and EMT. Moreover, one of the biological functions of ITGBL1 was to enhance the migration and invasion capabilities of MGC803 cells. In summary, these findings provided an insight that ITGBL1 served as an important diagnostic and prognostic tumor biomarker and a potential therapeutic target for GC, and hence was worth exploration.

\section{Materials And Methods}

\section{ITGBL1 expression in multiple databases}

To evaluate the expression level of ITGBL1 in somatic tumors, Oncomine Timer, and UALCAN public databases were used first. The Oncomine database is an online website containing Gene Expression Omnibus (GEO) and The Cancer Genome Atlas (TCGA) integration for endogenous mRNA levels. The 
Timer server is an integrated resource mainly used to analyze immune-infiltrating cells, but it also allows researchers to estimate the differential expression for genes of interest between tumor and adjacent normal tissues across diverse cancer types. The UALCAN database was used to compare the expression level of ITGBL1 not only in stomach adenocarcinoma and normal tissues but also in different clinical classification subgroups including cancer stages, sex, age, tumor grade, HP infection status, and nodal metastasis status. The distributions of gene expression levels are displayed using box plots.

\section{Survival analysis}

Kaplan-Meier Plotter is a website for online survival analysis involving a variety of tumors for GEO, European Genome-phenome Archive, and TCGA databases, which was used to evaluate the OS rate of gastric cancer tissues based on high-throughput sequencing data or gene chip.

\section{Prediction of association between TGBL1 and tumor-infiltrating immune cells}

The relationship between six immune infiltrates ( $B$ cells, CD4+ T cells, CD8+ T cells, neutrophils, macrophages, and dendritic cells) and ITGBL1 was estimated using the Timer algorithm. Besides, another immune-related web server, TISIDB, was used to analyze the association between the abundance of tumor-infiltrating lymphocytes (TILs) and expression of ITGBL1 by comparing the Spearman correlation between ITGBL1 expression and TILs in human cancers.

\section{Prediction of correlation between ITGBL1 and EMT-related factors}

Timer and Starbase web servers were used to assess the correlation between EMT-related factors and ITGBL1 expression level in gastric cancer tissues. The two databases contained the routine data analysis of the mRNA of any gene of interest in the TCGA, including differential expression, survival analysis, and co-expression. The degree of correlation was computed using purity-adjusted partial Spearman's rho value. The correlations of ITGBL1 with these factors was finally shown with scatter plots.

\section{Tissue specimens}

The study included 43 matched tumor samples from patients with surgically resected GC from the First Affiliated Hospital of Bengbu Medical College in 2017 for clinical indications. This study was authorized by the research medical ethics committee of the First Affiliated Hospital, Bengbu Medical College and Tongji Hospital, Tongji University. Each patient signed an informed consent form. All tissue specimens were pathologically confirmed as GC.

\section{Reverse transcription and quantitative real-time polymerase chain reaction}

Total RNA was extracted with TRIzol reagent (Life Technologies, CA, USA). Subsequently, cDNA was synthesized by reverse transcription with PrimeScript RT kits (Takara Biotechnology Co., Japan) following the manufacturer's protocol. Subsequently, generated CDNA was amplified with an SYBR PrimeScript RTPCR kit (Takara Biotechnology Co.) on an ABI 7900 Real-Time PCR system. The primers used in the study 
were as follows: ITGBL1, (sense) 5'-AGACCTACGACGGGAGCAC-3' and (antisense) 5'ACCTGCATTAGAGCAGATGATGT-3'; GAPDH, (sense) 5'-ATCACCATTGGCAATGAG-3' and (antisense) 5'AAGGTAGTTTCGTGGATG-3'. The $2^{\text {-comparative } C t}\left(2^{-\Delta \Delta C t}\right)$ formula was used to calculate the relative expression levels of ITGBL1, and GAPDH was normalized as an internal control.

\section{Immunohistochemical staining}

Tissues from patients with GC were fixed with $10 \%$ formalin for $48 \mathrm{~h}$ and then embedded with paraffin. Followed by deparaffinization and rehydration, the tissues were treated with $3 \% \mathrm{H}_{2} \mathrm{O}_{2}$ for 10 min prior to blocking in goat serum for $1 \mathrm{~h}$. Subsequently, a specific primary antibody ITGBL1 (1:100, Proteintech, China) was used to incubate the sections at $4^{\circ} \mathrm{C}$ overnight. Next, horseradish peroxidase-labeled secondary antibodies were used to incubate tissue sections for $1 \mathrm{~h}$ at $37^{\circ} \mathrm{C}$ and analyzed by the SABC method. The evaluation of color development results was performed as previously described [40].

\section{Cell culture}

Human normal gastric epithelial cell line GES-1 and GC cell lines SGC7901, BGC823, and MGC803 were provided by the Cell Division Center in Tongji Hospital of Tongji University, Shanghai, China. They were cultured in $90 \%$ DMEM (Gibco) with $10 \%$ fetal bovine serum (FBS, Gibco) at $37^{\circ} \mathrm{C}$ and incubated in a humidified $5 \% \mathrm{CO}_{2}$ atmosphere.

\section{Oligonucleotide transfection}

Further, $2.0 \times 10^{5}$ cells/well of MGC803 cell lines were seeded in a six-well plate prior to transfection. After reaching $50 \%$ fusion density, the cells were transfected with an ultimate concentration of $50 \mathrm{nM}$ of either negative control (NC) or ITGBL1-sh (targeted to interfere with ITGBL1) using Lipofectamine 2000 Reagent (Thermo Fisher Scientific, MA, USA). Three different oligonucleotide chains specifically targeting ITGBL1 were designed by Asia-Vector Biotechnology (Shanghai, China). The sequences were as follows: (sense) ITGBL1-sh1:5'-TGGGAAGTGTTACTGTGGA-3', (antisense) 5'-TCCACAGTAACACTTCCCA-3', (sense) ITGBL1-sh2: 5'- ACGATGAAACAGAAGAAAT-3', (antisense) 5'- ATTTCTTCTGTTTCATCGT-3', ITGBL1-sh3: (sense) 5'- GTGGACTTGTGTATGGTAA-3', (antisense) 5'- TTACCATACACAAGTCCAC-3'.

\section{Cell apoptosis assay}

Transfected cells were reseeded at a density of $5 \times 10^{5}$ cells/well onto six-well plates and collected with $0.25 \%$ trypsin without EDTA. Next, the collected cells were washed twice with cold PBS prior to resuspending with $1 \times$ binding buffer. After that, $5 \mu \mathrm{L}$ of Annexin V-PE and $10 \mu \mathrm{L}$ of AAD (Yeasen, China) were added to each cell suspension to stain cells and incubated for $30 \mathrm{~min}$. After adding $385 \mu \mathrm{L}$ of $1 \times$ Annexin-binding buffer, the cell apoptosis was assessed by flow cytometry.

\section{Transwell migration/invasion assay}


For assessing migration, the cells were cultured in the upper compartment of a Transwell chamber of an 8- $\mu$ m pore size insert (Corning, NY, USA) at a density of $1.5 \times 10^{4}$ cells/well in serum-free culture medium. For evaluating invasion, the cells were incubated in the upper chamber of the insert coated with Matrigel (Corning, NY, USA). The lower chambers of both experiments were treated with DMEM containing $20 \%$ FBS. Following $48 \mathrm{~h}$ incubation, the cells remaining on the upper compartment were wiped off with cotton swabs. The cells that migrated and invaded into the bottom chamber were stained with crystal violet for $30 \mathrm{~min}$. The results of the quantitative analysis were assessed using Image $\mathrm{J}$ software.

\section{Western blot analysis}

BCA protein assay was performed to determine protein concentrations. Equal amounts of protein lysates were segregated with $10 \%$ polyacrylamide gels (Bio-Rad, CA. USA) and transferred to $0.45 \mu \mathrm{m}$ nitrocellulose membranes (Bio-Rad). Then, 5\% BSA was used to block membranes for $1 \mathrm{~h}$ prior to incubation with primary antibodies: ITGBL1 (1:1000, Proteintech, China), Vimentin (1:1000, Cell Signaling, MA, USA), E-cadherin (1:1000, Proteintech, China), Snail (1:1000, Cell Signaling), TGF- $\beta 1$ (1:2000, Abcam, China), and GAPDH (1:2000, Proteintech, China) at $4^{\circ} \mathrm{C}$ overnight. Next, TBS containing $0.5 \%(w / v)$ Tween 20 buffer was used to wash membranes three times, and the membranes were incubated with the sheep anti-rabbit or sheep anti-mouse IgG secondary antibodies (1:10,000, LK, China) for $1 \mathrm{~h}$. Subsequently, the protein bands were detected using an ECL kit (Thermo Scientific) following the manufacturer's protocol. The GAPDH antibody served as a control.

\section{Statistical analysis}

Each experiment was performed at least in triplicate. Data were expressed as means \pm SEM. The discrepancies of grouping variables were analyzed using the Student $t$ test. All statistical analyses were performed using SPSS 20.0 , and $P<0.05$ indicated a statistically significant difference.

\section{Declarations}

\section{Acknowledgments}

Not applicable.

\section{Ethics approval and consent to participate}

Ethical approval was obtained by the research medical ethics committee of the First Affiliated Hospital, Bengbu Medical College and Tongji Hospital, Tongji University. Each patient signed an informed consent form.

\section{Consent for publication}

Not applicable. 
Availability of data and materials

The datasets used and/or analyzed during the current study are available from the corresponding author on reasonable request.

\section{Competing interests}

The authors declare no conflicts of interest.

\section{Funding}

This work was not supported.

\section{Authors' contributions}

Conceived and designed experiments: ZW and QW;

Performed experiments: ZW, LF, JZ and CG;

Analyzed experimental data: ZW, JZ and YG;

Technical assistance: RW and DM;

Wrote manuscript: ZW;

Study supervision: ZW.

\section{References}

1. Sung H, Ferlay J, Siegel RL, Laversanne M, Soerjomataram I, Jemal A, Bray F. Global Cancer Statistics 2020: GLOBOCAN Estimates of Incidence and Mortality Worldwide for 36 Cancers in 185 Countries. CA Cancer J Clin. 2021, 71(3): 209-49.

2. Karimi P, Islami F, Anandasabapathy S, Freedman ND, Kamangar F. Gastric Cancer: Descriptive Epidemiology, Risk Factors, Screening, and Prevention. Cancer Epidemiol Biomarkers Prev. 2014, 23(5): 700-13.

3. Van Cutsem E, Dicato M, Geva R, Arber N, Bang Y, Benson A, Cervantes A, Diaz-Rubio E, Ducreux M, Glynne-Jones $\mathrm{R}$, et al. The diagnosis and management of gastric cancer: expert discussion and recommendations from the 12th ESMO/World Congress on Gastrointestinal Cancer, Barcelona, 2010. Ann Oncol. 2011, 22:v1-v9.

4. Harris ES, Mclntyre TM, Prescott SM, Zimmerman GA. The Leukocyte Integrins. J Biol Chem. 2000, 275 (31): 23409-12.

5. Li F, Shang Y, Shi F, Zhang L, Yan J, Sun Q, She J. Expression of Integrin $\beta 6$ and HAX-1 Correlates with Aggressive Features and Poor Prognosis in Esophageal Squamous Cell Carcinoma. Cancer 
Manag Res. 2020, 12: 9599-608.

6. Fuentes P, Sesé M, Guijarro PJ, Emperador M, Sánchez-Redondo S, Peinado H, Hümmer S, Ramón y Cajal S. ITGB3-mediated uptake of small extracellular vesicles facilitates intercellular communication in breast cancer cells. Nature Communications. 2020, 11: 4261.

7. Huaman J, Ogunwobi 00. Circulating Tumor Cell Migration Requires Fibronectin Acting through Integrin B1 or SLUG. Cells. 2020, 9(7): 1594.

8. Berg RW, Leung E, Gough S, Morris C, Yao W, Wang S, Ni J, Krissansen GW. Cloning and Characterization of a Novel Integrin-Related cDNA Coding for the Protein TIED ("Ten $\beta$ Integrin EGFlike Repeat Domains") That Maps to Chromosome Band 13q33: A Divergent Stand-Alone Integrin Stalk Structure. Genomics. 1999, 56: 169-78.

9. Qiu X, Feng J, Qiu J, Liu L, Xie Y, Zhang Y, Liu J, Zhao Q. ITGBL1 promotes migration, invasion and predicts a poor prognosis in colorectal cancer. Biomed Pharmacother. 2018, 104: 172-80.

10. Huang W, Yu D, Wang M, Han Y, Lin J, Wei D, Cai J, Li B, Chen P, Zhang X. ITGBL1 promotes cell migration and invasion through stimulating the TGF-beta signalling pathway in hepatocellular carcinoma. Cell Prolif. 2020, 53(7): e12836.

11. Gan X, Liu Z, Tong B, Zhou J. Epigenetic downregulated ITGBL1 promotes non-small cell lung cancer cell invasion through Wnt/PCP signaling. Tumor Biology. 2015, 37(2): 1663-9.

12. Li R, Zhuang C, Jiang S, Du N, Zhao W, Tu L, Cao H, Zhang Z, Chen X. ITGBL1 Predicts a Poor Prognosis and Correlates EMT Phenotype in Gastric Cancer. Journal of Cancer. 2017, 8(18): 3764-73.

13. Li T, Fan J, Wang B, Traugh N, Chen Q, Liu JS, Li B, Liu XS. TIMER: A Web Server for Comprehensive Analysis of Tumor-Infiltrating Immune Cells. Cancer Res. 2017, 77(21):e108-10.

14. Rohdes RD, Yu J, Shanker K, Deshpande N, Varambally R, Ghosh D, Barrette T, Pandey A, Chinnaiyan AM. ONCOMINE: A Cancer Microarray Database and Integrated Data-Mining Platform. Neoplasia. 2004, 6:1-6

15. Chandrashekar DS, Bashel B, Balasubramanya SAH, Creighton CJ, Ponce-Rodriguez I, Chakravarthi B, Varambally S. UALCAN: A Portal for Facilitating Tumor Subgroup Gene Expression and Survival Analyses. Neoplasia. 2017, 19(8): 649-58.

16. Gyorffy B, Lanczky A, Eklund AC, Denkert C, Budczies J, Li Q, Szallasi Z. An online survival analysis tool to rapidly assess the effect of 22,277 genes on breast cancer prognosis using microarray data of 1,809 patients. Breast Cancer Res Treat. 2010, 123(3):725-31.

17. Gao J, Aksoy BA, Dogrusoz U, Dresdner G, Gross B, Sumer SO, Sun Y, Jacobsen A, Sinha R, Larsson E, et al. Integrative Analysis of Complex Cancer Genomics and Clinical Profiles Using the cBioPortal. Science Signaling. 2013, 6(269): pl1-pl1.

18. Ru B, Wong CN, Tong Y, Zhong J, Zhong S, Wu W, Chu K, Wong C, Lau C, Chen I, et al. TISIDB: an integrated repository portal for tumor-immune system interactions. Bioinformatics. 2019, 35(20): 4200-2.

19. Li J, Liu S, Zhou H, Qu L, Yang J. StarBase v2.0: decoding miRNA-ceRNA, miRNA-ncRNA and proteinRNA interaction networks from large-scale CLIP-Seq data. Nucleic Acids Res. 2014, 42(Database 
issue):D92-7.

20. Kong J, Tian H, Zhang F, Zhang Z, Li J, Liu X, Li X, Liu J, Li X, Jin D, et al. Extracellular vesicles of carcinoma-associated fibroblasts creates a pre-metastatic niche in the lung through activating fibroblasts. Molecular Cancer. 2019, 18: 175.

21. Taeyeon K, Khurshid A, Sibhghatulla S, Tasleem JA, Myung-Gi S, Ju LE, Inho C. Dermatopontin in Skeletal Muscle Extracellular Matrix Regulates Myogenesis. Cells. 2019, 8(4): 332.

22. Qi L, Song F, Ding Y. Regulatory Mechanism of ITGBL1 in the Metastasis of Colorectal Cancer. Frontiers in Oncology. 2020, 10: 259.

23. Ji Q, Zhou L, Sui H, Yang L, Wu X, Song Q, Jia R, Li R, Sun J, Wang Z, et al. Primary tumors release ITGBL1-rich extracellular vesicles to promote distal metastatic tumor growth through fibroblast-niche formation. Nature Communications. 2020, 11: 1211.

24. Lian X, Ma J, Zhou J, Zhang T, Wu D, Deng Z, Zhang Z, Li X, He P, Yan Y, et al. Hypermethylation of ITGBL1 is associated with poor prognosis in acute myeloid leukemia. Journal of Cellular Physiology. 2018, 234(6): 9438-46.

25. Li S, Defeng W, Xiaotian L, Lingling Z, Hui Z, Yingjie Z. Extracellular matrix protein ITGBL1 promotes ovarian cancer cell migration and adhesion through WntPCP signaling and FAKSRC. Biomedicine \& Pharmacotherapy. 2016, 81:145-51.

26. Liu X, Wu J, Zhang D, Bing Z, Tian J, Ni M, Zhang X, Meng Z, Liu S. Identification of Potential Key Genes Associated With the Pathogenesis and Prognosis of Gastric Cancer Based on Integrated Bioinformatics Analysis. Frontiers in Genetics. 2018, 9: 265.

27. Gu Y, Chen Y, Jin K, Cao Y, Liu X, Lv K, He X, Lin C, Liu H, Li H, et al. Intratumoral CD103(+)CD4(+) T cell infiltration defines immunoevasive contexture and poor clinical outcomes in gastric cancer patients. Oncoimmunology. 2020, 9(1):1844402.

28. Zhang $X, X u$ W. Neutrophils diminish T-cell immunity to foster gastric cancer progression: the role of GM-CSF/PD-L1/PD-1 signalling pathway. Gut. 2017, 66(11):1878-80.

29. Cheli Y, Tulic MK, El Hachem N, Nottet N, Jacquel A, Gesson M, Strub T, Bille K, Picard-Gauci A, Montaudie $\mathrm{H}$, et al. ITGBL1 is a new immunomodulator that favors development of melanoma tumors by inhibiting natural killer cells cytotoxicity. Mol Cancer. 2021, 20:12.

30. Chae YK, Chang S, Ko T, Anker J, Agte S, lams W, Choi WM, Lee K, Cruz M. Epithelial-mesenchymal transition (EMT) signature is inversely associated with T-cell infiltration in non-small cell lung cancer (NSCLC). Scientific Reports. 2018, 8: 2918.

31. Huang B, Sun L, Cao J, Zhang Y, Wu Q, Zhang J, Ge Y, Fu LIU, Wang Z. Downregulation of the GnT-V gene inhibits metastasis and invasion of BGC823 gastric cancer cells. Oncology Reports. 2013, 29(6): 2392-400.

32. Yang S, Liu Y, Li M-Y, Ng CSH, Yang S-I, Wang S, Zou C, Dong Y, Du J, Long X, et al. FOXP3 promotes tumor growth and metastasis by activating Wnt/ $\beta$-catenin signaling pathway and EMT in non-small cell lung cancer. Molecular Cancer. 2017, 16: 124. 
33. Choi S, Yu J, Park A, Dubon MJ, Do J, Kim Y, Nam D, Noh J, Park KS. BMP-4 enhances epithelial mesenchymal transition and cancer stem cell properties of breast cancer cells via Notch signaling. Sci Rep. 2019, 9(1): 11724.

34. Jiao D, Chen J, Li Y, Tang X, Wang J, Xu W, Song J, Li Y, Tao H, Chen Q. MiR-1-3p and miR-206 sensitizes HGF-induced gefitinib-resistant human lung cancer cells through inhibition of c-Met signalling and EMT. J Cell Mol Med. 2018, 22(7): 3526-36.

35. Tripathi V, Shin JH, Stuelten CH, Zhang YE. TGF-beta-induced alternative splicing of TAK1 promotes EMT and drug resistance. Oncogene. 2019, 38(17): 3185-200.

36. Wang M, Gong Q, Zhang J, Chen L, Zhang Z, Lu L, Yu D, Han Y, Zhang D, Chen P, et al.

Characterization of gene expression profiles in HBV-related liver fibrosis patients and identification of ITGBL1 as a key regulator of fibrogenesis. Sci Rep. 2017, 7:43446.

37. Takatoshi M, Toshiaki I, Naoki T, Yasuhide Y, Masamichi Y, Tatsuyuki K, Hiroyuki U, Ajay G.

Transcriptomic expression profiling identifies ITGBL1, an epithelial to mesenchymal transition (EMT)associated gene, is a promising recurrence prediction biomarker in colorectal cancer. Molecular Cancer. 2019, 18:19.

38. Li X, Du X, Li D, Kong P, Sun Y, Liu P, Wang Q, Feng Y. ITGBL1 Is a Runx2 Transcriptional Target and Promotes Breast Cancer Bone Metastasis by Activating the TGF Signaling Pathway. Cancer Res. 2015; 75 (16): 3302-13.

39. Li W, Li S, Yand J, Cui C, Yu M, Zhang Y. ITGBL1 promotes EMT, invasion and migration by activating NF-KB signaling pathway in prostate cancer. Oncotargets Ther. 2019; 12: 3753-63.

40. Dong S, Wang Z, Huang B, Zhang J, Ge Y, Fan Q, Wang Z. Bioinformatics insight into glycosyltransferase gene expression in gastric cancer: POFUT1 is a potential biomarker. Biochem Bioph Res Co. 2017; 483: 171-7.

\section{Figures}


A

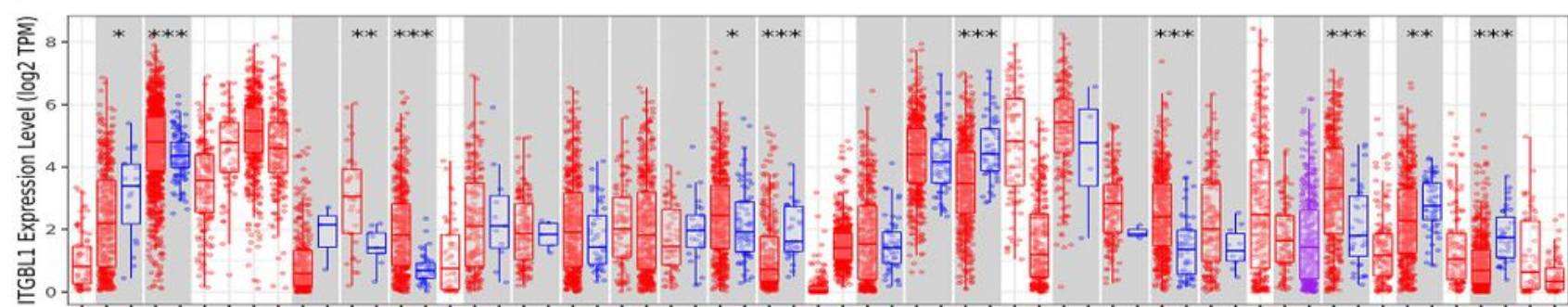

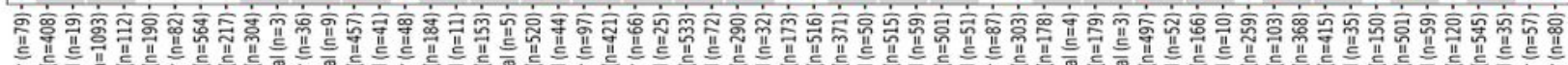

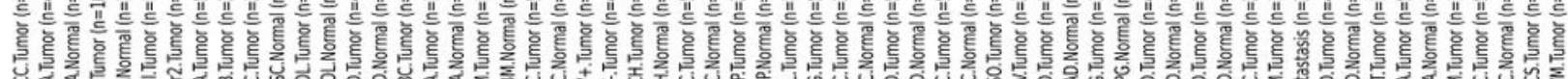

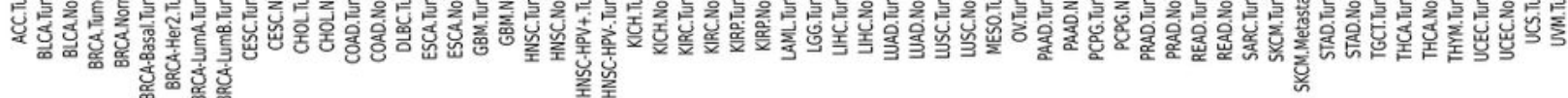

B

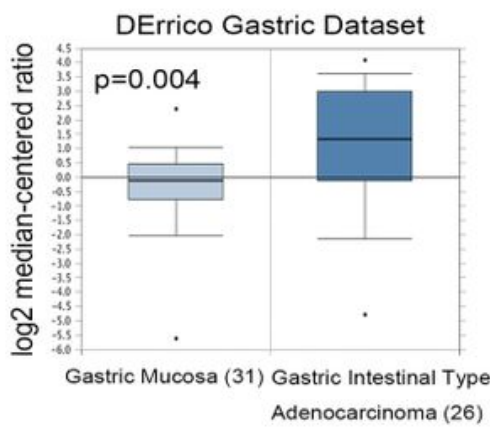

D

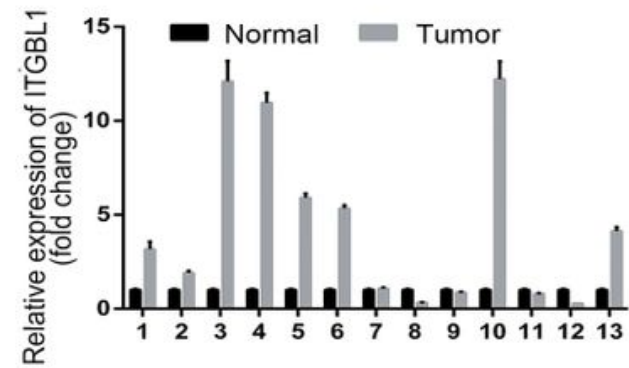

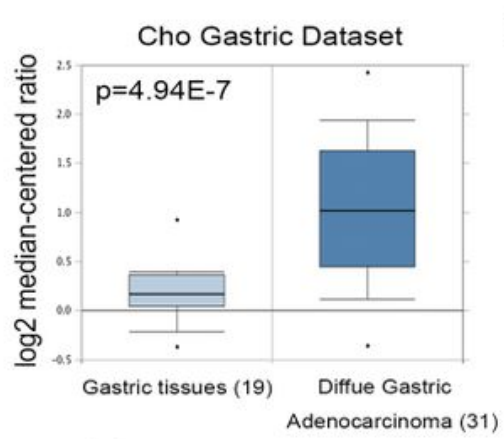

$E$

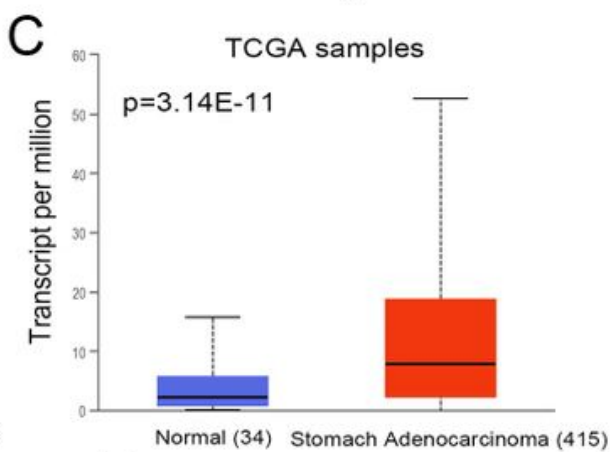

$\mathrm{F}$
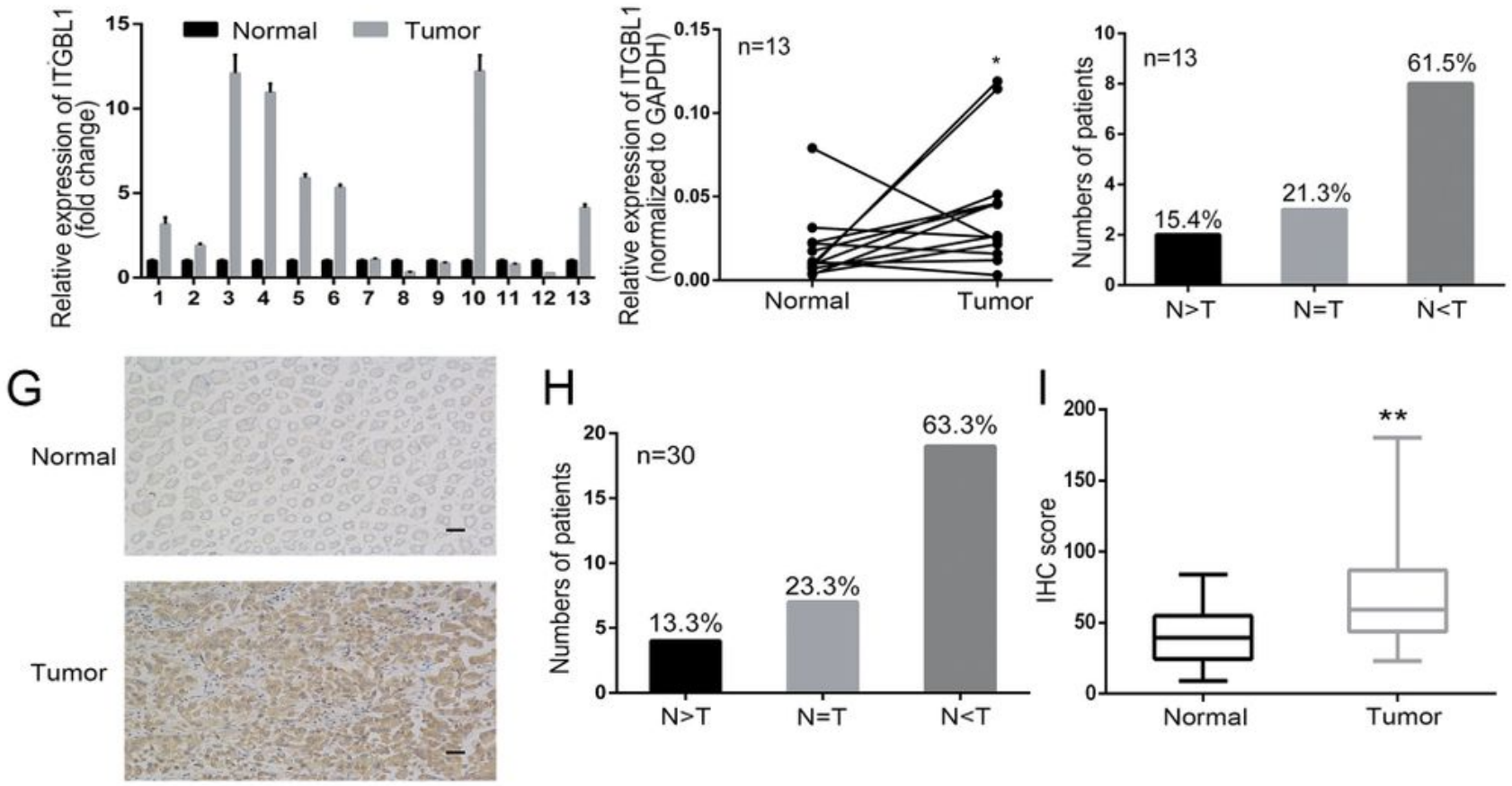

$\mathrm{H}$

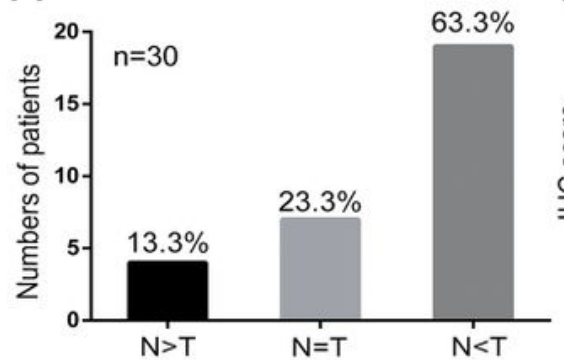

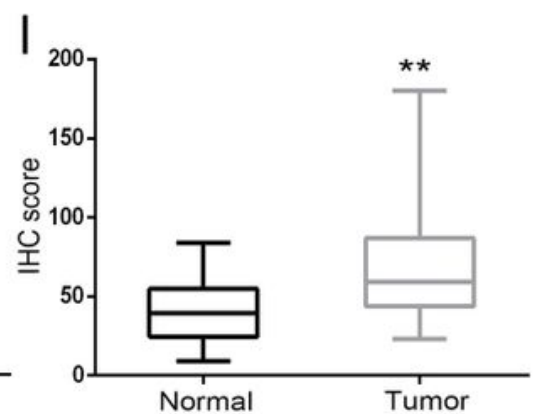

\section{Figure 1}

ITGBL1 levels in GC tissues. (A) mRNA expression of ITGBL1 of different tumors in the Timer database. (B) mRNA levels of ITGBL1 significantly increased in GC tissues compared with normal tissues based on the Oncomine database. (C) In UALCAN, ITGBL1 mRNA expression was upregulated in stomach adenocarcinoma tissues compared with normal tissues. (D-F) QRT-PCR was conducted to detect ITGBL1 mRNA expression in 13 GC samples as fold change (D) and normalized to GAPDH expression (E). Results 
showed that $61.5 \%(8 / 13)$ of patients with GC had high expression of ITGBL1 (F). (G-I) Immunohistochemical staining of ITGBL1 for 30 paired patients with GC. Representative images of normal and tumor tissues are shown; scale bar: $10 \mu \mathrm{m}(\mathrm{G}) ; 63.3 \%$ of patients with ITGBL1-positive expression in GC tissues $(\mathrm{H})$. Results of ITGBL1 IHC scores in GC compared with paired nontumor tissues (I). ${ }^{*} \mathrm{P}<0.05,{ }^{*} \mathrm{P}<0.01$.
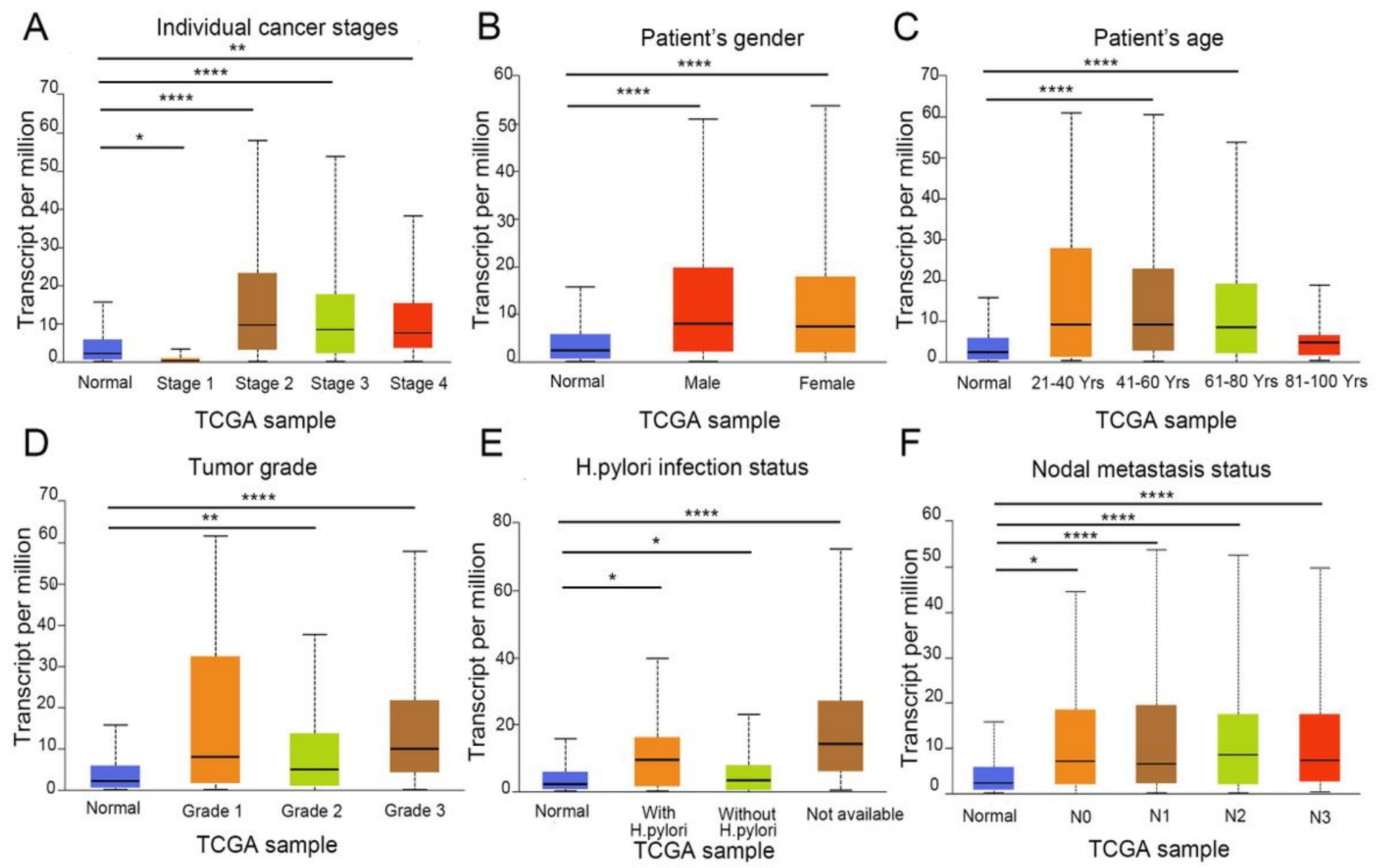

Figure 2

Clinical factors associated with ITGBL1 expression. ITGBL1 expression in subgroups of patients with GC based on cancer stage, sex, age, tumor grade, $\mathrm{H}$. pylori infection status, and nodal metastasis status. ${ }^{*} \mathrm{P}<$ $0.05, * \star \mathrm{P}<0.01, * \star \star * \mathrm{P}<0.0001$ 
A

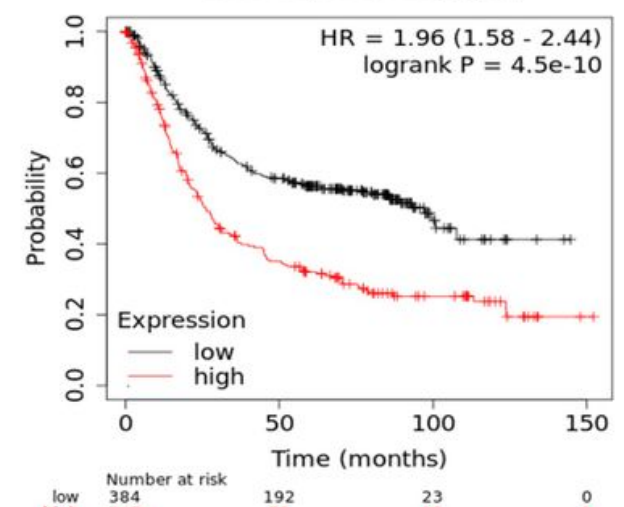

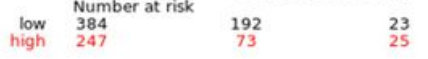

$\mathrm{D}$

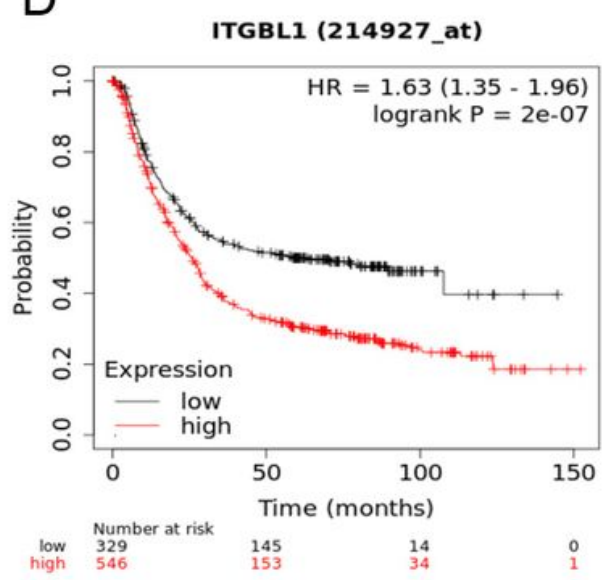

B

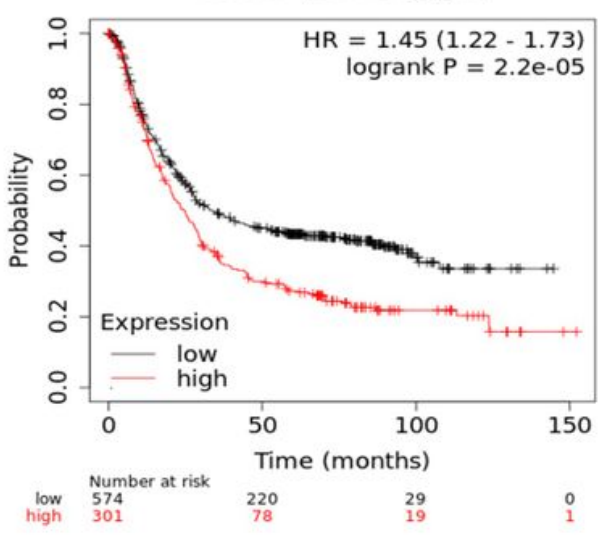

$E$

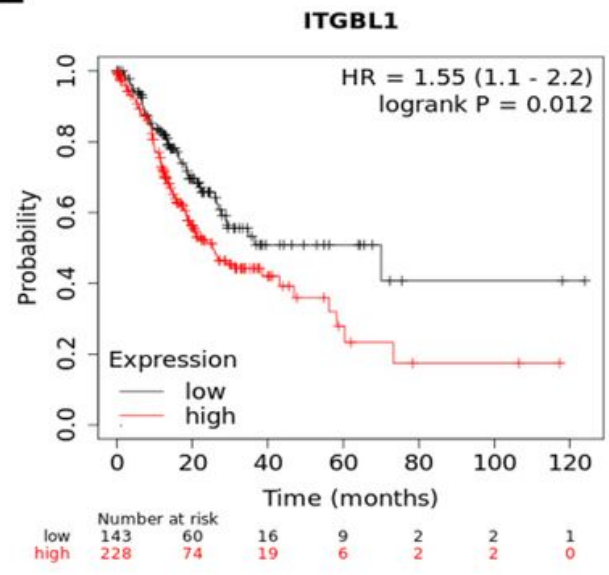

C

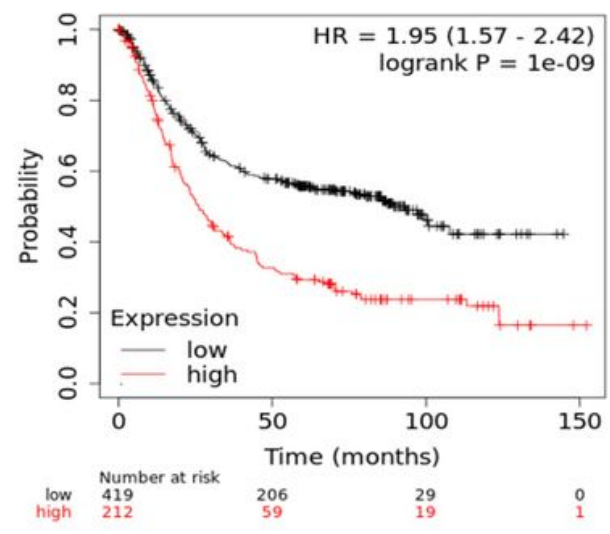

$\mathrm{F}$

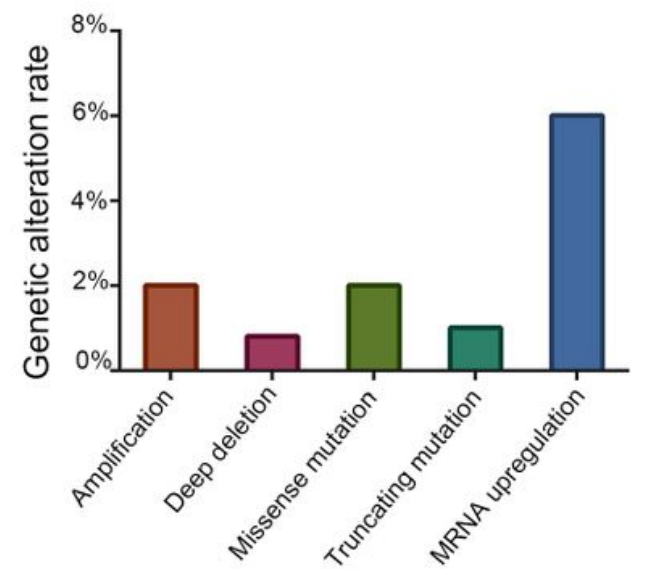

\section{Figure 3}

Prognostic value of ITGBL1. (A-D) Overall survival of ITGBL1 in different gene chips based on the Kaplan-Meier Plotter. (E) In the Kaplan-Meier Plotter, the prognosis of ITGBL1 is shown with RNA sequencing. (F) Genomic alterations of ITGBL1 were analyzed using cBioPortal in GC. 

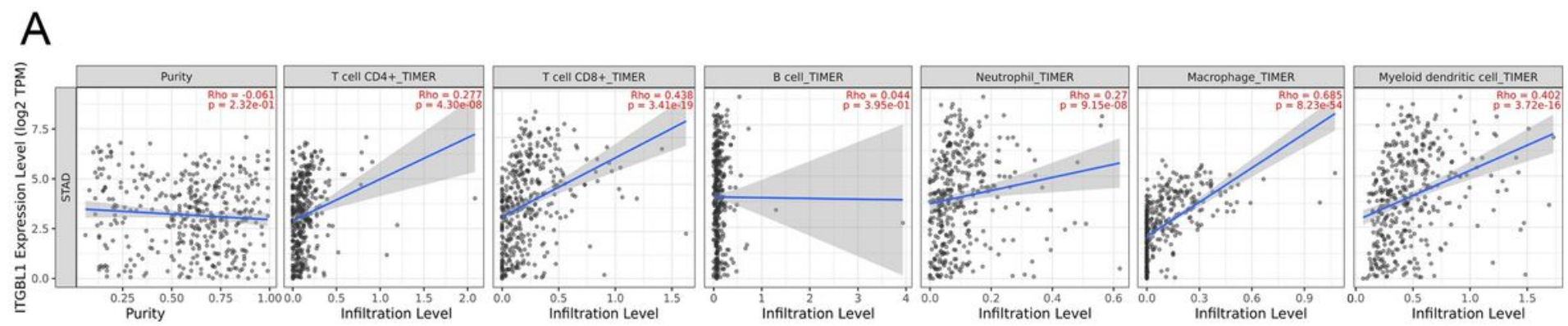

$\mathrm{B}$
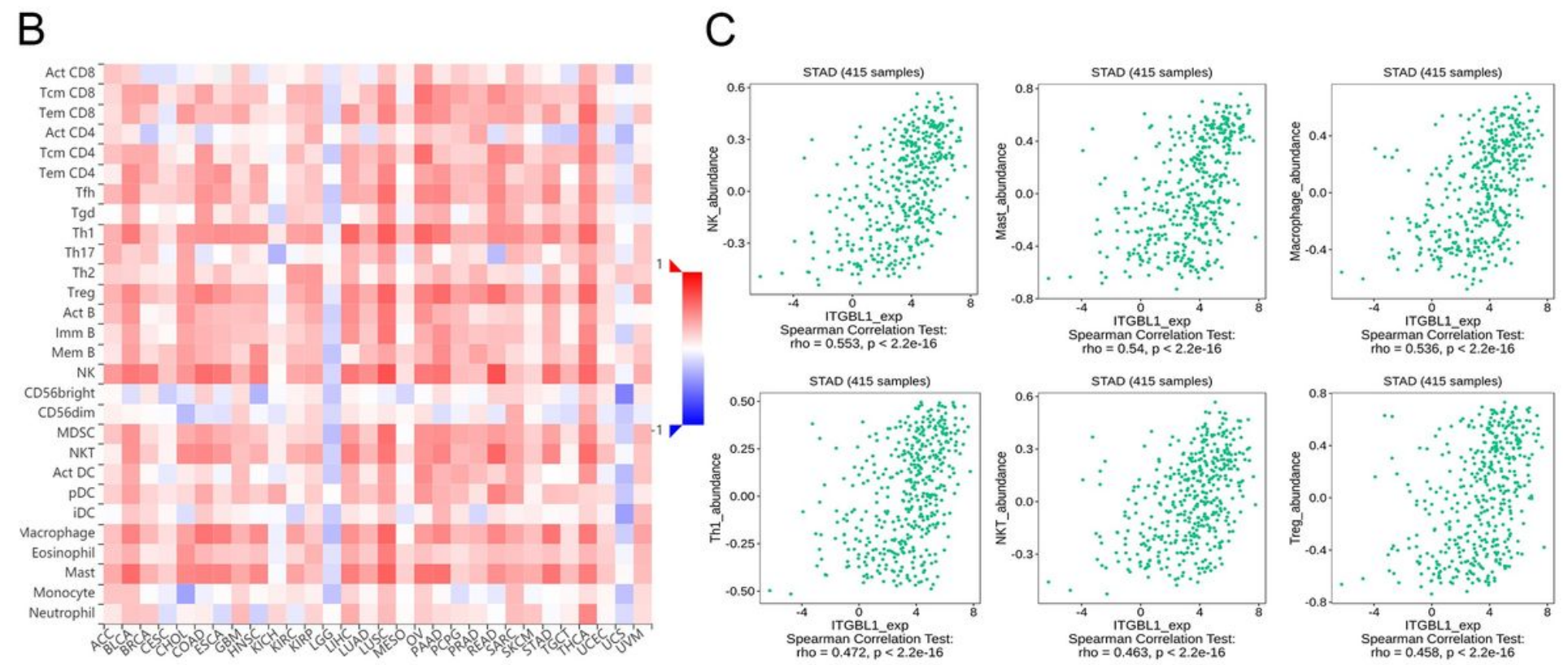

\section{Figure 4}

Association of ITGBL1 with immune-infiltrating cells. (A) Timer database was used to predict the correlation between ITGBL1 and different immune cells (CD4+ T cells, CD8+ cells, neutrophils, macrophages, B cells, and myeloid dendritic cells). (B) Hot plot showed the relations between the abundance of immune-related signatures of 28 TILs and ITGBL1 expression in diverse tumors. (C) Top six immune cells with a strong correlation with ITGBL1. 

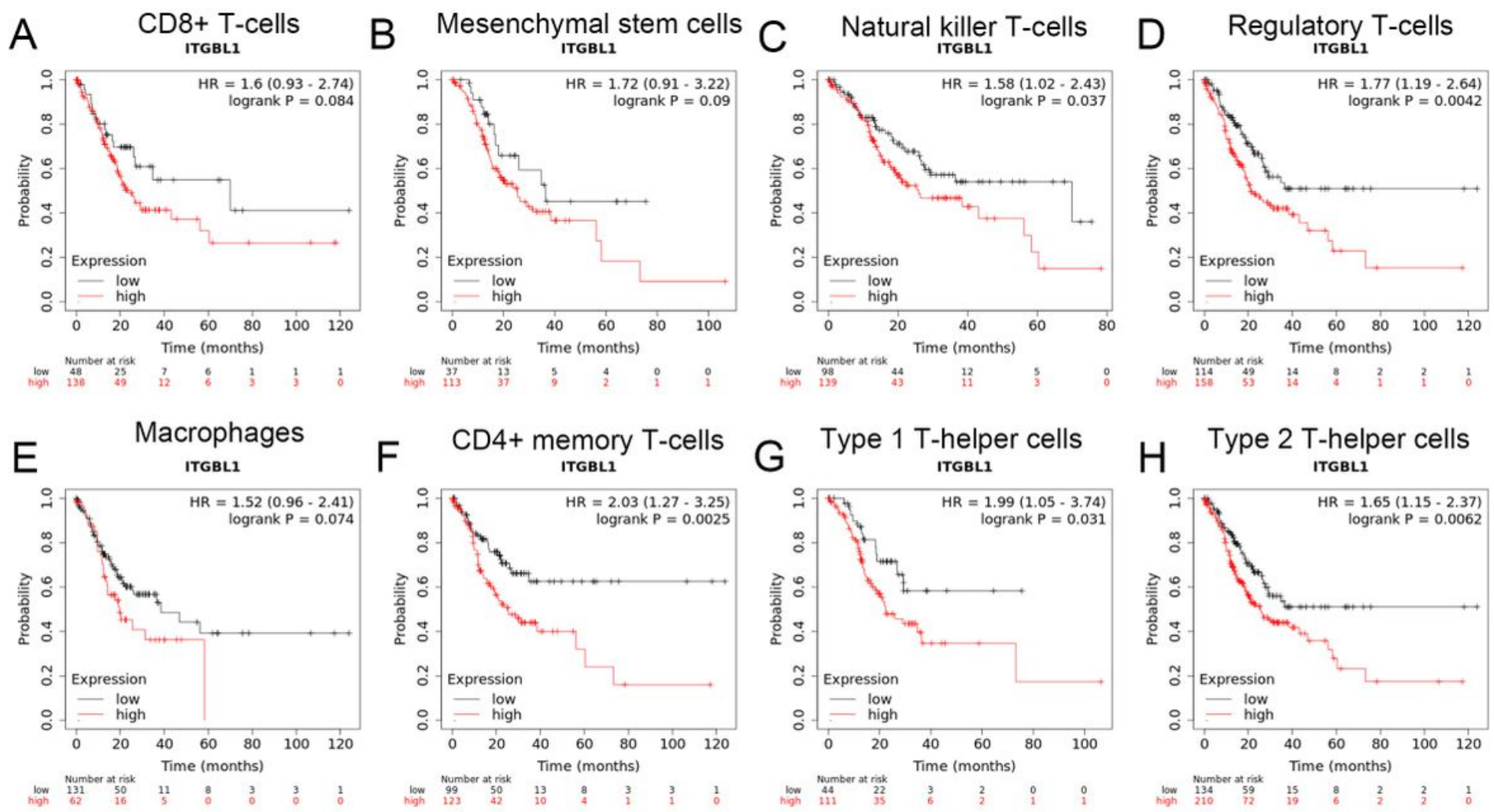

\section{Figure 5}

Prognosis of ITGBL1 in enriched natural killer T cells, regulatory T cells, CD4+ memory T cells, Type $1 \mathrm{~T}$ helper cells, Type 2 T helper cells, CD8+ T cells, mesenchymal stem cells, and macrophages. 


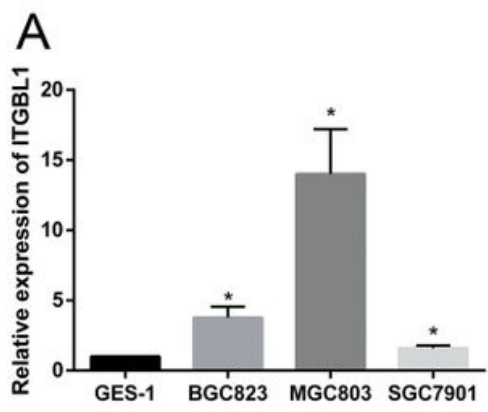

B

C
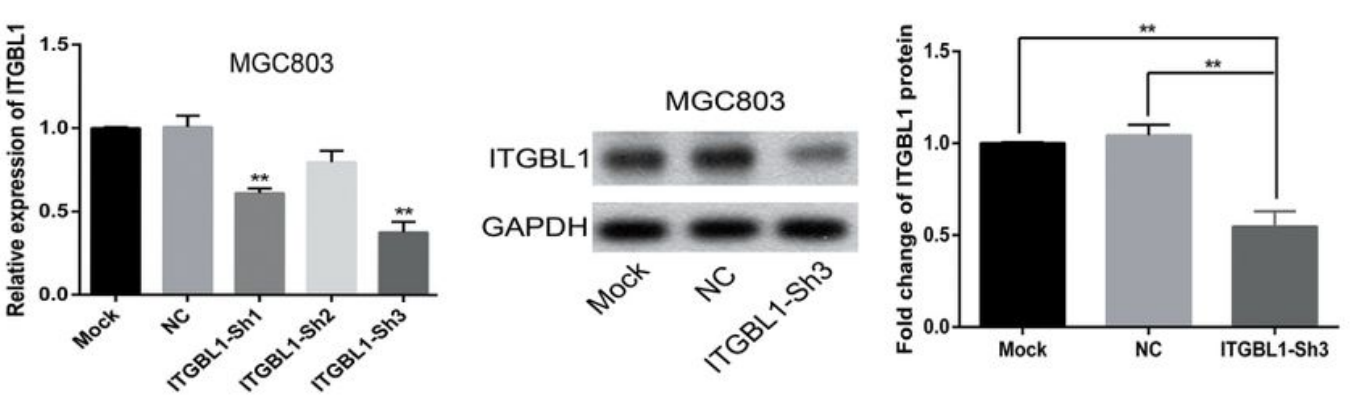

D
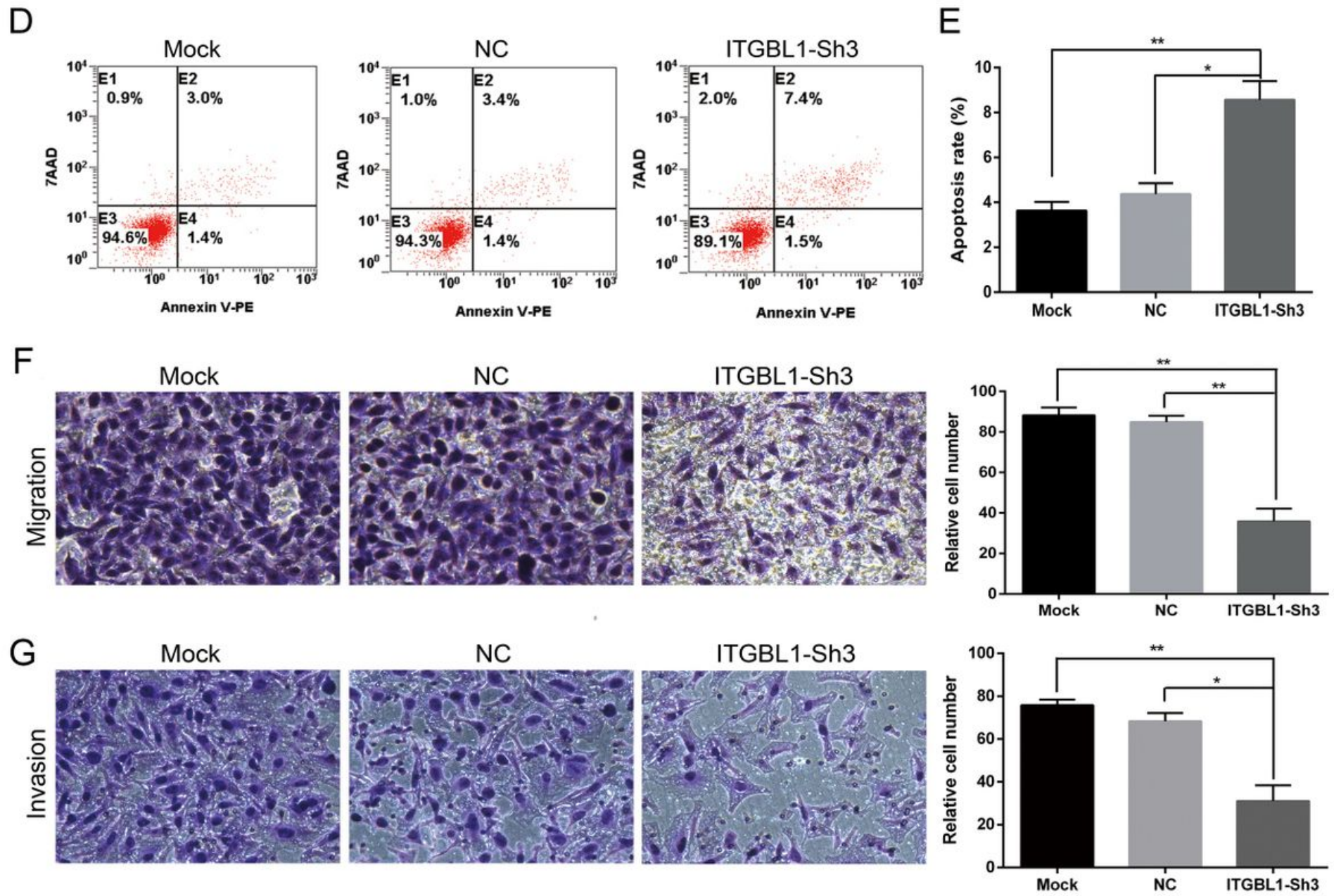

Figure 6

Expression of ITGBL1 in GC cells and silencing of ITGBL1 promoted apoptosis and inhibited the migration and invasion of MGC803 cells. (A) Results of qRT-PCR for ITGBL1 mRNA expression in GC cell lines relative to GES-1. (B and C) Results of qRT-PCR and Western blot for the silencing of ITGBL1. GAPDH was used as an internal control. (D and E) Representative images and quantification results for flow cytometry to evaluate apoptosis induction by ITGBL1 in MGC803 cells transfected with or without ITGBL1-Sh3. (F) Representative images for Transwell assay and quantification results of Transwell migration. Migrated cells were analyzed using ImageJ. (G) Representative images of Transwell invasion 
assay and quantitative analysis of invasive cells using Image $J$. The results showed that the knockdown of ITGBL1 suppressed cell migration and invasion. ${ }^{*} \mathrm{P}<0.05, * * \mathrm{P}<0.01$.
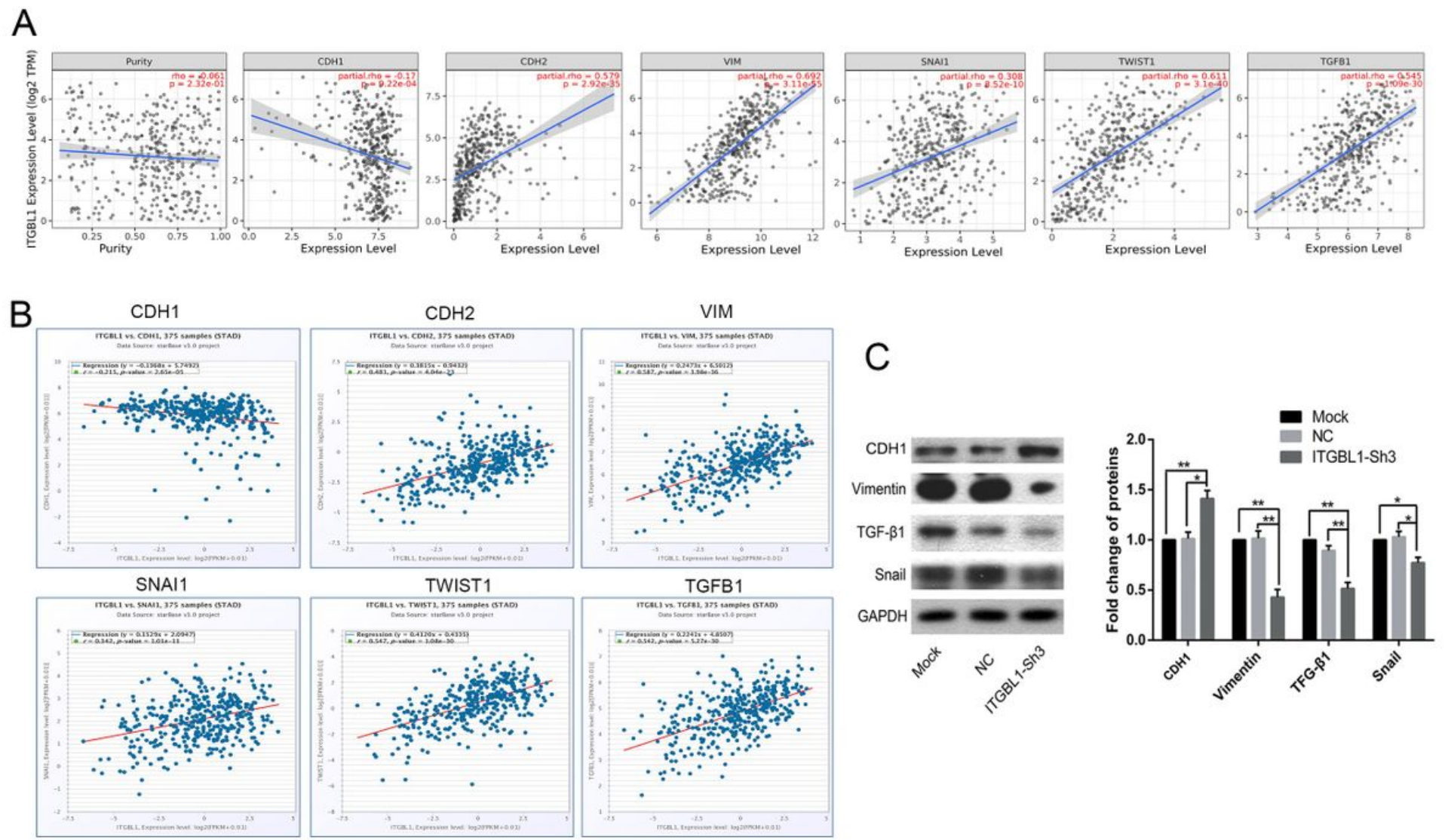

Figure 7

ITGBL1 was involved in the EMT process in MGC803 cells. (A) Online Timer database was used to explore the correlation between the expression of ITGBL1 and EMT-related factors. (B) Starbase further predicted the relationship of TIGBL1 and CDH1, CDH2, Vim, Snai1, Twist1, and TGF- $\beta 1$. (C) Representative images and quantitative Western blot analysis of the expression of $\mathrm{CDH} 1$, Vimentin, Snail, and TGF- $\beta 1$ proteins in MGC803 cells treated with mock or NC or silencing of ITGBL1. ${ }^{\star} P<0.05, * \star P<$ 0.01 . 